\title{
On the interpretation of lateral manganin gauge stress measurements in polymers
}

\author{
Gareth J. Appleby-Thomas, ${ }^{\text {a) }}$ Paul J. Hazell, ${ }^{\text {b) }}$ James M. Wilgeroth, and David C. Wood \\ Cranfield Defence and Security, Cranfield University, Shrivenham, Swindon SN6 8LA, \\ United Kingdom
}

(Received 9 April 2010; accepted 8 June 2010; published online 12 August 2010)

\begin{abstract}
Encapsulated wire-element stress gauges enable changes in lateral stress during shock loading to be directly monitored. However, there is substantial debate with regards to interpretation of observed changes in stress behind the shock front; a phenomenon attributed both to changes in material strength and shock-dispersion within the gauge-encapsulation. Here, a pair of novel techniques which both modify or remove the embedding medium where such stress gauges are placed within target materials have been used to try and inform this debate. The behavior of three polymeric materials of differing complexity was considered, namely polystyrene, the commercially important resin transfer moulding (RTM) 6 resin and a commercially available fat (lard). Comparison to the response of embedded gauges has suggested a possible slight decrease in the absolute magnitude of stress. However, changing the encapsulation has no detectable effect on the gradient behind the shock in such polymeric systems. (C) 2010 American Institute of Physics. [doi:10.1063/1.3460812]
\end{abstract}

\section{INTRODUCTION}

It is well known that materials behave in a significantly different manner under high strain-rates/pressures-e.g., shock loading - compared to quasistatic/low strain-rate conditions. Material properties at elevated strain-rates may be experimentally investigated using a variety of techniques. However, for strain-rates in the regime $10^{6}-10^{8} / \mathrm{s}$ the most common laboratory-scale approach is the plate-impact technique. A useful summary of this approach together with comparison to other high strain-rate deformation techniques is provided by Field et al. $;^{1}$ essentially, a launch system (typically a compressed-gas or explosively-driven gun) is used to impact a target material with a projectile/flyer plate at elevated velocities/pressures, imparting a shock wave into the target. During such experiments all surfaces perpendicular to the impact axis are carefully kept flat/parallel to a tolerance of $<5 \mu \mathrm{m}$. Under such conditions inertial confinement ensures a one-dimensional (1D) shock in the center of the target up until the point where release waves from either the flyer rear-surface or target edge catch up with the main shock. ${ }^{2,3}$ Consequently, careful design allows experiments to be conducted entirely in a 1D state of strain. Shock propagation may be monitored via a variety of techniques including interferometeric monitoring of the target rear surface and insertion of embedded pressure gauges into the material flow. Five discrete parameters describe such a shock: shock velocity $\left(\mathrm{U}_{\mathrm{S}}\right)$; particle or mass velocity $\left(\mathrm{u}_{\mathrm{P}}\right.$; the continuum velocity of the material behind the shock front); pressure (equivalent to the longitudinal stress, $\sigma_{\mathrm{X}}$, established within the material over the shock duration where material strength is negligible); density, and; internal energy. However, these parameters are linked by a series of conservation relations

\footnotetext{
a)Electronic mail: g.applebythomas@cranfield.ac.uk.

b) Also at Institute of Shock Physics, Royal School of Mines, Prince Consort Road, Imperial College London, London, SW7 2BP, UK.
}

known as the Rankine-Hugoniot conservation equations. These equations mean that a relationship between any two of these parameters, known as a Hugoniot equation-of-state, is sufficient to uniquely define a shock. ${ }^{4}$

In plate-impact experiments longitudinal (in-plane/Hugoniot- $\sigma_{\mathrm{X}}$ ) stress behavior behind a shock is normally a function of the input pulse. For example, if a square-wave is introduced then a shock with a rectangular form will result. However, while plate-impacts are 1D, inertial confinement will lead to establishment of a lateral stress $\left(\sigma_{\mathrm{Y}}\right)$. Unlike the longitudinal stress case, measurements of lateral stress via encapsulated lateral manganin gauges often show a change in gradient behind the shock..$^{2,3,5-11}$ The established explanation for this phenomena is based on Eq. (1); i.e., that the change in gradient represents a change in shear strength $(\tau)$ behind the shock. ${ }^{2,3,5-9}$ Assuming constant $\sigma_{\mathrm{X}}$, any change in $\sigma_{\mathrm{Y}}$ would, therefore, represent a change in $\tau$, e.g., a decrease in $\sigma_{\mathrm{Y}}$ would correspond to strengthening within the target. In most cases appropriate microstructural mechanisms are identified to explain any observed changes in strength. An increase in strength is often observed in polymeric materials and is typically linked to compression of long-chain molecules. ${ }^{3}$ The converse effect, namely, an increase in lateral stress/a reduction in $\tau$ (weakening) behind the shock has been observed in bcc materials. An example of weakening behavior behind the shock occurs in tungsten, where it was attributed to a combination of the impedance of dislocation formation, motion, and storage by a high PeierlsNabarro stress in addition to initiation of cracks along grain boundaries. ${ }^{12}$

$$
\tau=\frac{\sigma_{\mathrm{X}}-\sigma_{\mathrm{Y}}}{2} .
$$

As an alternative, Winter and Harris ${ }^{10}$ and Winter et al. ${ }^{11}$ suggested that perceived changes in strength behind the shock might be linked to differential target and encapsulation 
shock velocities. Shock propagation was simulated through (a) a so-called matrix material (the target) and (b) a matrix material with a central fluid layer representing an encapsulated gauge. This work showed significant modification of the shock front due to different fluid layer/matrix velocities. A faster shock velocity in the fluid layer led to a continual rise in lateral gauge stress following shock arrival. Conversely, a faster matrix shock speed resulted in an initial ramp to a peak (possibly linked to the formation of a Mach stem behind the slower moving shock in the encapsulation) followed by a steady decrease in magnitude behind the front. Experiments were conducted by Winter et al. ${ }^{11}$ on tantalum and steel targets with the aim of confirming this effect by monitoring recession velocities during plate-impact tests both on, and a finite distance from, the impact axis. The targets employed sandwiched a lateral gauge encapsulation proxy (a $100 \mu \mathrm{m}$ thick Mylar central layer). Observed significant differences in the recession velocities, predicted numerically, ${ }^{10}$ were attributed to modification of the shock by the central encapsulation.

Both explanations for the observed behavior of lateral gauge traces seemed plausible. Consequently, a series of experiments designed to investigate the effect of modification of the encapsulation layer on lateral stress were undertaken. Recent work involving lateral gauge encapsulation within the commercially important resin transfer moulding (RTM) 6 epoxy resin showed a gradient despite similar resin/interlayer wave speeds. ${ }^{13}$ This suggested that lateral gauges appear to detect genuine changes in material properties behind the shock. ${ }^{3}$ However, this work did not consider potential effects of a significant missmatch in target/encapsulation impedance. Here, a series of lateral gauge shots on readily available materials employing a variety of different forms of central gauge region were undertaken in an attempt to address this issue. These approaches were: (1) standard epoxy-resin encapsulation; (2) a dry-joint, with the gauge physically clamped between the two target material halves, and; (3) direct suspension of a lateral gauge in a castable biological adipose material. The dry-joint case was designed to create an air gap ahead of the gauge element which would rapidly damp-out the incident shock in the encapsulation region. It was reasoned that this would provide a significant target/ gauge encapsulation impedance missmatch. Further, prevention of shock propagation in the interlayer implied that a dry-mounted lateral gauge should only detect changes in lateral stress resulting from the inertial confinement of the target, with no contribution from the absent interlayer. The use of a castable material in the third case was designed to remove the interlayer entirely. A similar approach was adopted in recent work with the polymeric material Sylguard by Millett et al. ${ }^{14}$ An observed decrease in lateral stress behind incident shocks in Sylguard was attributed to a strengthening phenomenon (steric interference). In this work material was cast directly around the gauge, itself encased between supporting sheets of $50 \mu \mathrm{m}$ thick Mylar with, in two of three tests undertaken, no encapsulation ahead of the gauge element. Given this approach it was reasoned that any response would be primarily attributable to the material instead of being a function of the nature of gauge encapsulation.
The materials chosen for the first two tests were the simple (structure) thermoplastic Polystyrene ${ }^{15,16}$ and more complex thermosetting resin RTM 6., ${ }^{3,13}$ Primarily due to their low densities and ability to be cast into near net-shape final structures, polymers represent an important class of materials. They have been widely adopted in applications as diverse as aerospace composites and the matrix materials used to bind energetic crystals together in explosive compositions. In such applications components can potentially be exposed to shock loading; i.e., loading at a sufficiently high strain-rate/pressure to overcome material shear strength (resistance to shear) allowing hydrodynamic (fluid-like) flow to occur. Consequently, knowledge of polymeric material behavior in such high strain-rate regimes is desirable. Further, given previous experimental evidence of changes in lateral stress behind the shock, these two materials seemed an appropriate initial point for further investigation of the behavior of lateral gauges. For the third test a commercially available fat (manufactured by Matthews Foods plc and retailed by the Co-operative ${ }^{\circledR}$ Food Group under the brand name "Fresh Fields Lard") was employed. In addition to its ready availability, this material was chosen for two key reasons: (i) as it was castable at a relatively low temperature $\left(50-60{ }^{\circ} \mathrm{C}\right.$; with melting occurring at $35-40{ }^{\circ} \mathrm{C}$ ), and (ii) due to the complex long-chain structural nature of the glycerol units/ attached fatty acids $^{17}$ (which were expected to lead to behavior analogous to a complex polymer like RTM 6 under shock-loading).

\section{EXPERIMENTAL}

Lateral stress behavior was investigated via a series of plate-impact experiments ${ }^{2,3}$ conducted on a $50 \mathrm{~mm}$ bore, $5 \mathrm{~m}$ barrel, single-stage gas-gun. ${ }^{18}$ The temporal evolution of lateral stress both at, and following, shock arrival was monitored using embedded Manganin lateral stress gauges of type J2M-SS-580SF-025, manufactured by Vishay MicroMeasurements, USA. Two different approaches were employed for target preparation dependant on whether glued/ dry-jointed targets or a cast target were employed. However, all tests were based on a standard experimental procedure with regards to the use of lateral gauges previously discussed in the literature. ${ }^{2,3}$ A generic lateral gauge target arrangement is shown in Fig. 1. Lateral gauges were introduced 2-4 mm from the impact face for the polystyrene and RTM 6 targets and at 4-6 $\mathrm{mm}$ for the cast adipose material. Gauge calibration was based on the assumption that the thin-foil lateral embedded gauges reached a state of strain-equilibrium with the surrounding target material. ${ }^{19}$ In addition, the calibration technique employed incorporated both the elastic-plastic behavior of manganin ${ }^{20}$ and its pressure-dependent behavior at stresses below its elastic limit. ${ }^{21}$ Finally, key material data required for lateral gauge calibration (densities and longitudinal/shear wave sound speeds) were taken from the literature for polystyrene ${ }^{15,16,22}$ and measured using a Micrometrics AccuPyc 1330 gas pycnometer (density) and a Panametrics 5077PR pulse receiver in the pulse-echo configuration (sound speeds) for RTM 6. ${ }^{3,13}$ In the case of the adipose material employed to directly encapsulate gauges a density 


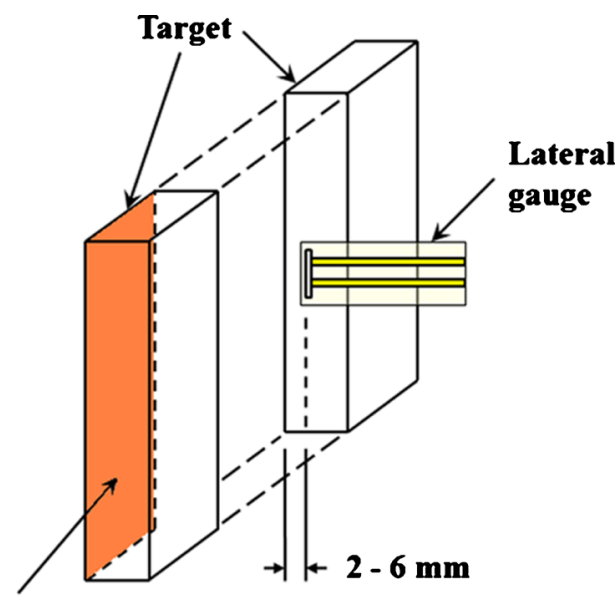

Impact face (hidden)

FIG. 1. (Color online) Generic lateral gauge arrangement.

of $0.945 \pm 0.001 \mathrm{~g} / \mathrm{cm}^{3}$ and longitudinal sound speed of $1.51 \pm 0.01 \mathrm{~mm} / \mu$ s was measured using similar techniques. Unfortunately, due to the attenuating nature of the fat it proved difficult to directly measure a shear sound wave speed. However, as only the general form of the resultant traces was of interest the decision was taken to present lateral gauge traces as raw voltages, rather than calibrated stress data. Consequently no attempts are made to draw more than qualitative conclusions from these results.

Where glued-joints were required the gauge was encapsulated between two target halves whose faces were finished to a roughness of $<5 \mu \mathrm{m}$ (Fig. 1). Care was taken to ensure that the gauge element was positioned both at a measured position from $(2-4 \mathrm{~mm})$, and parallel to, the target impact face. At this point the insulated legs of the gauge were temporarily tagged to the rear-surface of one target half using superglue. A slow cure epoxy (Loctite 0151 HYSOL $^{\circledR}$ Epoxi-Patch $^{\circledR}$ ) allowed to set for $>24 \mathrm{~h}$ was subsequently employed to adhere target halves using a clamping arrangement which ensured a flat/parallel bond. For the "dry" joints the two target halves were clamped using an identical ar- rangemen but were instead secured together without an epoxy interlayer via the (visible) rear-surface. In the case of cast targets the encapsulating arrangement shown schematically in Fig. 2 was employed. This arrangement comprised a $1 \mathrm{~mm}$ thick $\mathrm{Cu}$ plate adhered to a $10 \mathrm{~mm}$ thick ring along its edge with two holes (one to allow lard to be cast in situ; the second to provide a path for degassed air to escape during casting). The stiffness of the lateral gauge was enhanced by encapsulation in $50 \mathrm{~mm}$ thick Mylar. Two different configurations were considered. In the first, as shown in Fig. 2, care was taken to ensure that the Mylar did not protrude further than the front edge of as-manufactured lateral gauge. This meant that only cast material lay between the front of the gauge and the cover plate. With the second geometry the encapsulating Mylar was allowed to run ahead of the gauge such that it eventually lay flush with the $\mathrm{Cu}$ cover plate. In both cases the resultant arrangement was clamped between, and protruded through (to a desired/measured distance), two machined sections of polymethylmethacrylate (PMMA) subsequently employed as the rear surface of the capsule. By ensuring that the gauge was perpendicular to the machined PMMA surface and that the gauge element was parallel to the impact face good alignment was maintained.

Assembled targets were attached to a target ring, itself retained within a sacrificial barrel extension incorporating a series of conducting pins. These pins, when shorted by an incident projectile, provided a measure of the impact velocity. Just before impact, a pair of conducting pins was used to trigger a $1 \mathrm{GHz}$ oscilloscope employed to record stress information. This arrangement with a generic target is shown schematically in Fig. 3.

\section{RESULTS AND DISCUSSION}

\section{A. Glued/dry-joints}

The response of lateral gauges embedded in polystyrene and RTM 6 targets was investigated via a total of nine experiments (four and five tests, respectively). As the features of lateral gauge response, rather than particular stress

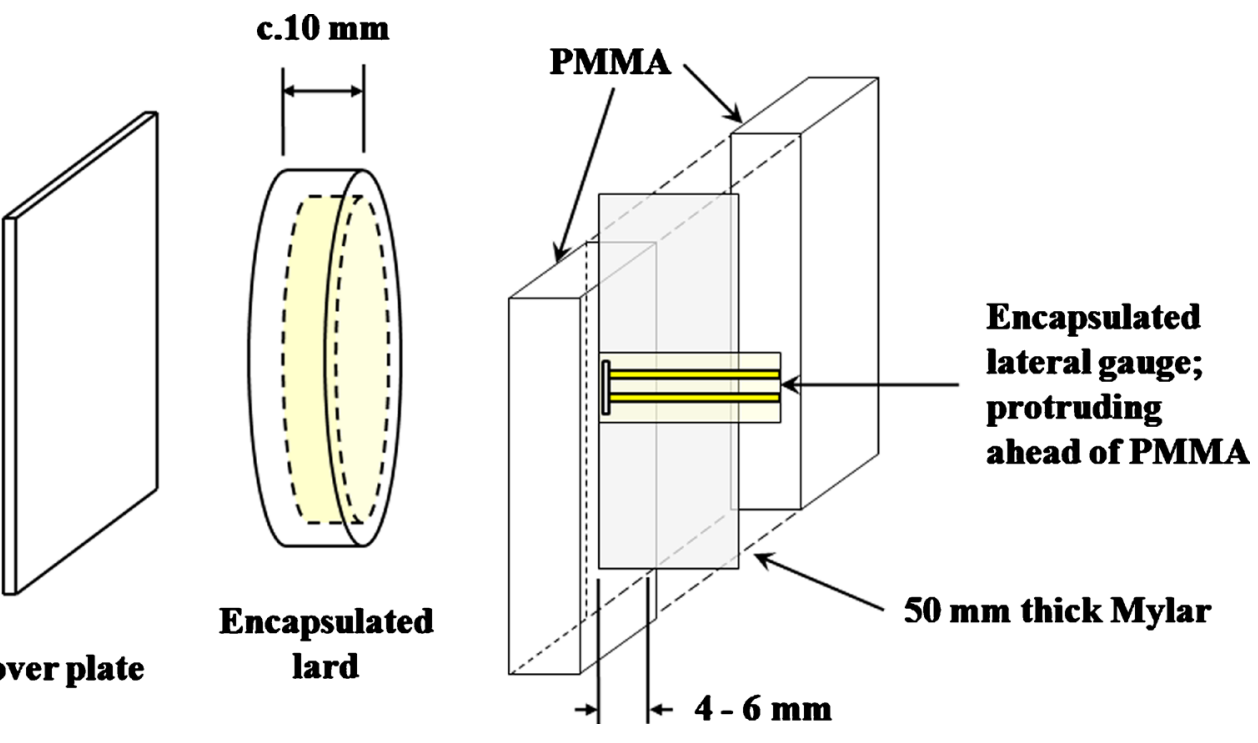

FIG. 2. (Color online) Schematic illustration of key elements of lard-encapsulation system (casting holes not-shown). 


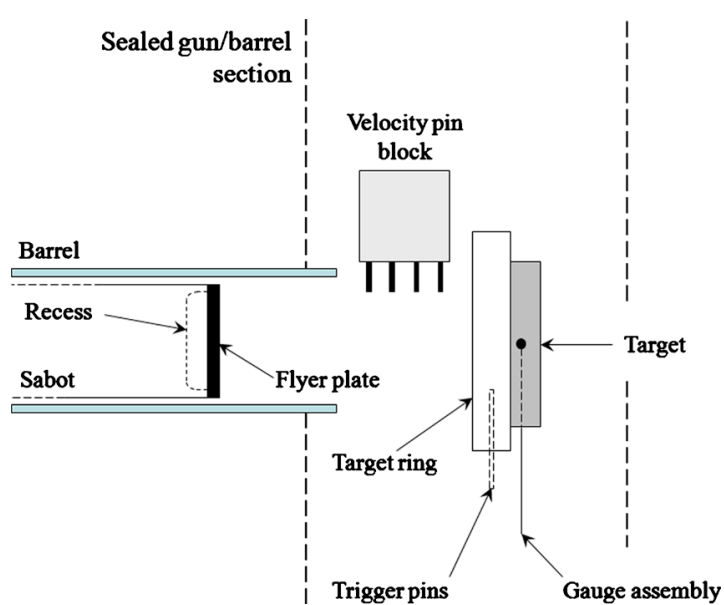

FIG. 3. (Color online) Schematic illustration of a typical plate-impact target experimental setup.

achieved, were of interest all shots were undertaken using broadly similar experimental conditions. Copper (a well characterized material ${ }^{22}$ ) was chosen for the flyer material. Flyer thicknesses were kept at $5 \mathrm{~mm}$ for all of the polystyrene shots and three of the five RTM 6 shots, with the remainder using $10 \mathrm{~mm}$ thick flyers. Pressures in the gas-gun breech were approximately constant for all shots. Consequently, while with $5 \mathrm{~mm} \mathrm{Cu}$ flyers impact velocities of c. $430 \mathrm{~m} / \mathrm{s}$ were achieved, with the heavier $10 \mathrm{~mm}$ thick flyers lower impact velocities of c. $410 \mathrm{~m} / \mathrm{s}$ were recorded. Experimental configurations employed along with measured gradients in the subsequently recorded gauge traces (Figs. 4 and 5 for polystyrene and RTM 6 , respectively) are presented in Table I.

Resultant lateral gauge traces based on the experiments detailed in Table I are presented in Figs. 4 and 5 for polystyrene and RTM 6, respectively. With both materials, a rapid rise on shock arrival is followed by a slowly decreasing stress plateau behind a peak. This is ended by a drastic reduction in stress due to the arrival of release waves from the rear of the flyer. A small plateau following initial shock arrival, not present with gauges at $4 \mathrm{~mm}$, was apparent where gauges were positioned $2 \mathrm{~mm}$ from the impact face. However, the key features in traces with a $2 \mathrm{~mm}$ gauge (i.e., magnitude and stress gradient behind the shock) rapidly met

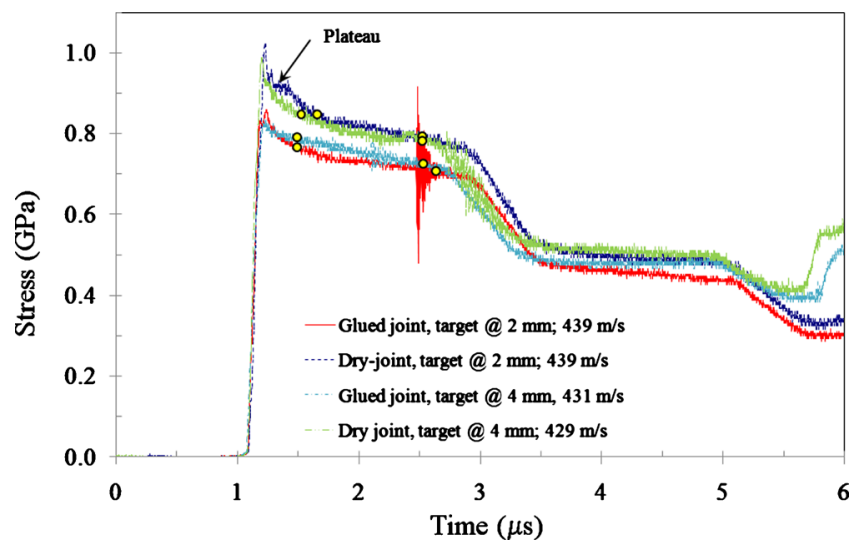

FIG. 4. (Color online) Polystyrene lateral gauge traces $(5 \mathrm{~mm}$ thick $\mathrm{Cu}$ flyer).

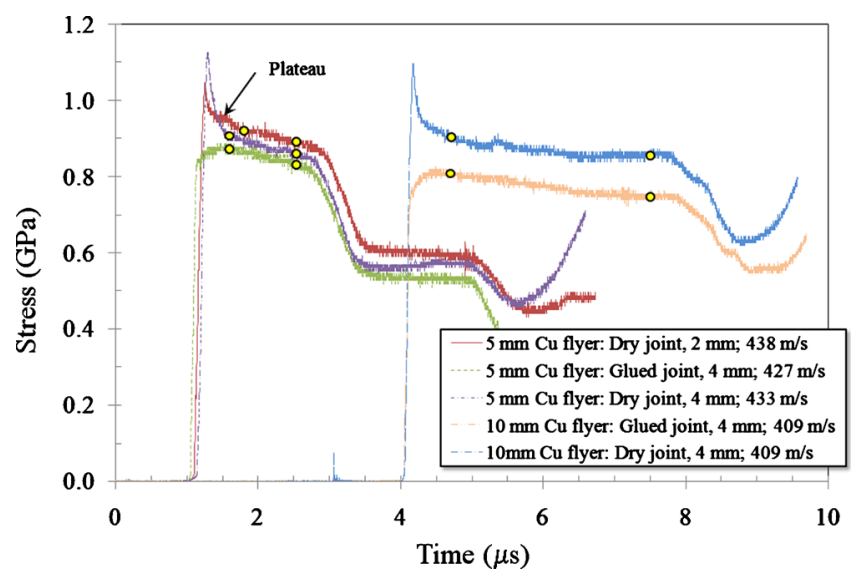

FIG. 5. (Color online) RTM 6 lateral gauge traces

equivalent traces with the gauge mounted at $4 \mathrm{~mm}$. Consequently, this initial disturbance had no long term effect on gauge response. Following this initial plateau (where present), gradients were measured behind the shock for both materials. The magnitude of these stress gradients was found to be different for polystyrene and RTM 6. However, when each material was considered in isolation, the nature of lateral gauge encapsulation employed was found to have no discernable effect on the measured gradient.

As discussed, two competing theories have been put forward to explain the observed behavior behind the shock. These are: (i) strengthening/an increase in $\tau$ following Eq. (1) and (ii) shock front dispersion due to a slower shock velocity in the central encapsulating layer than in the target material. This latter effect would lead to formation of a Mach stem behind the gauge resulting in an overshoot in lateral stress and a subsequent decay in stress behind the shock. ${ }^{10,11}$ Yellow markers setting out the extent of gradients behind the shock front are shown in Figs. 4 and 5, with gradients measured between these points detailed in Table I. These markers were deliberately positioned away from any initial post-rise gauge effects. All the polystyrene gradients exhibited similar magnitudes of c. $0.06 \mathrm{GPa} / \mu \mathrm{s}$. As discussed, the air gap between the target impact face and the embedded gauge in dry-joint tests was expected to rapidly damp-out the shock in the gauge encapsulation region. Therefore, if the observed

TABLE I. Experimental configurations-modified encapsulation tests.

\begin{tabular}{lcccc}
\hline \hline & $\begin{array}{c}\text { Gauge position/ } \\
\text { joint-type } \\
(\mathrm{mm})\end{array}$ & $\begin{array}{c}\text { Impact } \\
\text { velocity } \\
(\mathrm{m} / \mathrm{s})\end{array}$ & $\begin{array}{c}\text { Flyer } \\
\text { thickness } \\
(\mathrm{mm})\end{array}$ & $\begin{array}{c}\text { Gradient behind } \\
\text { the shock between } \\
\text { yellow markers } \\
\text { in Figs. 4 and 5 } \\
(\mathrm{GPa} / \mu \mathrm{s})\end{array}$ \\
\hline Material & 2/glued & 439 & 5 & -0.055 \\
Polystyrene & 4/glued & 439 & 5 & -0.064 \\
Polystyrene & 2/dry & 431 & 5 & -0.061 \\
Polystyrene & 4/dry & 429 & 5 & -0.060 \\
RTM 6 & 4/glued & 427 & 5 & -0.039 \\
RTM 6 & 2/dry & 438 & 5 & -0.041 \\
RTM 6 & 4/dry & 433 & 5 & -0.047 \\
RTM 6 & 4/glued & 409 & 10 & -0.019 \\
RTM 6 & 4/dry & 409 & 10 & -0.021 \\
\hline
\end{tabular}


negative gradient behind the shock was attributable to a slower shock velocity in the central encapsulating layer, a steeper gradient in the dry-joint case might reasonably be expected; whereas similar gradients are observed here. Consequently, the observed similar gradients behind the shock for polystyrene, independent of encapsulation technique, shown in Table I seem to imply that lateral gauge behavior is linked to a material rather than geometrical/target-assembly based response.

Similar trends to those observed in the polystyrene lateral response are apparent with the RTM 6 data, although with two notable differences. First, despite very similar impact conditions, the magnitude of the gradient, where a $5 \mathrm{~mm}$ flyer was employed, was c. $17 \%$ less than in polystyrene. This change in magnitude appears to counter the suggestion made by Winter and Harris ${ }^{10}$ that, because Rosenberg and Partom ${ }^{19}$ based their initial gauge calibration on systems where the gauge encapsulation and target impedances closely matched, the interpretation of gauge response will primarily pick up effects due to the encapsulation. Instead, simply moving between two materials with relatively similar densities $\left(1.03 \pm 0.02\right.$ and $1.141 \pm 0.001 \mathrm{~g} / \mathrm{cm}^{3}$ for polystyrene and RTM 6, respectively) and shock Hugoniots leads to a consistent and measurable difference in gradient. Winter and Harris ${ }^{10}$ and Winter et al. ${ }^{11}$ suggest such a steeper gradient might be attributed to a faster shock speed in polystyrene than RTM 6. However, very similar shock velocity-particle velocity $\left(\mathrm{U}_{\mathrm{S}}-\mathrm{u}_{\mathrm{P}}\right)$ Hugoniot relationships of $\mathrm{U}_{\mathrm{S}}=2.65$ $+1.55 \mathrm{u}_{\mathrm{P}}$ and $2.21+1.84 \mathrm{u}_{\mathrm{P}}$ have been measured for RTM $6^{13}$ and polystyrene ${ }^{15}$ respectively. Consequently, not only are shock speeds under near-identical loading conditions very similar but for $u_{\mathrm{P}}<1.5 \mathrm{~mm} / \mu \mathrm{s}$, shock velocities will be nominally greater in RTM 6 than polystyrene. Further, the suggestion that a lagging shock leading to a Mach stem in the encapsulation region governs the formation of a negative gradient ${ }^{10,11}$ does not appear reasonable in this case as RTM 6 is an epoxy resin system. Consequently, because there is known to be a high degree of similarity in $\mathrm{U}_{\mathrm{S}}-\mathrm{u}_{\mathrm{P}}$ Hugoniot relationships for different epoxies, ${ }^{13}$ shock velocities will be comparable in both RTM 6 and an epoxy-filled interlayer for given impact conditions. This means that Mach stem formation due to shock dispersion is unlikely to occur in the epoxy-centered RTM 6 (glued-joint) targets considered here.

This result appears to be further evidence that lateral gauges are detecting a true material rather than gauge-based response. Other results that confirm this interpretation are: (i) the presence of observed negative gradients in RTM 6 behind the shock despite the similar shock impedance of the material and the gauge-encapsulating epoxy; (ii) the lack of a link between the observed gradients and the nature of encapsulation, and; (iii) the difference in the magnitude of the observed gradients compared to those seen with polystyrene.

A second difference between the polystyrene and RTM 6 data from Table $\mathrm{I}$ is the reduction in the magnitude of the measured gradient from c. 0.02 to c. $0.04 \mathrm{GPa} / \mu$ s when a 10 rather than $5 \mathrm{~mm}$ thick flyer was employed. This is caused by the observed flattening of the stress behind the shock in Fig. 5. The models employed by Winter et al. ${ }^{11}$ do suggest establishment of such a far-field constant stress. However, the near-identical gradients apparent in the glued and dry-joint 10 mm flyer RTM 6 shots considered here make it reasonable to suggest that this flattening of the curve behind the shock represents the end of the original strengthening mechanism governing the gradient. The recent work by Appleby-Thomas et $a l^{3}$ involving longitudinal gauges mounted either side of an RTM 6 target containing an encapsulated lateral gauge suggested a similar conclusion; e.g., no significant evidence of shock dispersion was found despite the presence of a negative gradient behind the shock in the lateral gauge trace. Combined with the lack of a change in gradient observed in Figs. 4 and 5 when the interlayer is substantially modified (e.g., the encapsulation is replaced with a dry-joint/air gap), such results give further credence to the suggestion that, at least in the polymeric materials considered here, the nature of the interlayer has no significant effect on any changes in lateral stress observed behind the shock. This strongly suggests that such changes represent a true material response to the applied shock-favoring the interpretation that the gradient represents a change in material strength.

Tentative evidence was apparent of a link between the form of the gauge response (e.g., the nature of the overshoot following shock arrival and the final stress obtained) and the nature of the encapsulating layer. A lower overall stress behind the shock, combined with a gentler initial ramp-up, is observed in both Fig. 4 and Fig. 5 where an encapsulating interlayer was employed. The decrease in stress where a glued rather than dry-interlayer was employed in otherwise identical experiments was typically 10\%-13\% (except in the $4 \mathrm{~mm}$ gauge position $5 \mathrm{~mm} \mathrm{Cu}$ flyer RTM 6 case, where a lower difference of $2.1 \%$ was initially attributed to slightly different impact velocities). At first glance, this relatively consistent increase across two different materials appeared to suggest a mechanical or geometrical—rather than materialresponse. However, when the data presented in Figs. 4 and 5 was converted from measured voltages accorded to established procedures ${ }^{2,3,19-21}$ it was also recentered to start at zero volts. The vertical offset introduced into the original data was at most $0.01 \mathrm{~V}$. However, in the polymeric materials considered here this small error was a significant fraction of recorded voltages for the impact conditions set out in Table I. The error of $\pm 0.01 \mathrm{~V}$ compared to typically measured voltages of just $0.11-0.12 \mathrm{~V}$ for both polystyrene and RTM 6; e.g., equivalent to up to $9 \%$ of the measured voltage. This was a similar ratio to the observed difference in stress discussed above; in-line with this result, when the effects of these initial offsets are included the stress magnitudes in the polystyrene (but interestingly not the RTM 6) traces are found to be essentially coincident. Consequently, more work will be required before the differences in stress magnitude can be categorically confirmed as a true gauge (rather than data reduction) response. Nevertheless, the overall form of the recorded lateral gauge traces, and in particular the observed changes in initial overshoot behavior, are unaffected by initial recentring of the recorded gauge data.

The larger overshoots in stress following shock arrival observed in the dry-joint cases for both polystyrene and RTM 6 appear to be consistent with the stress perturbations predicted for the slower encapsulation shock velocity case by 


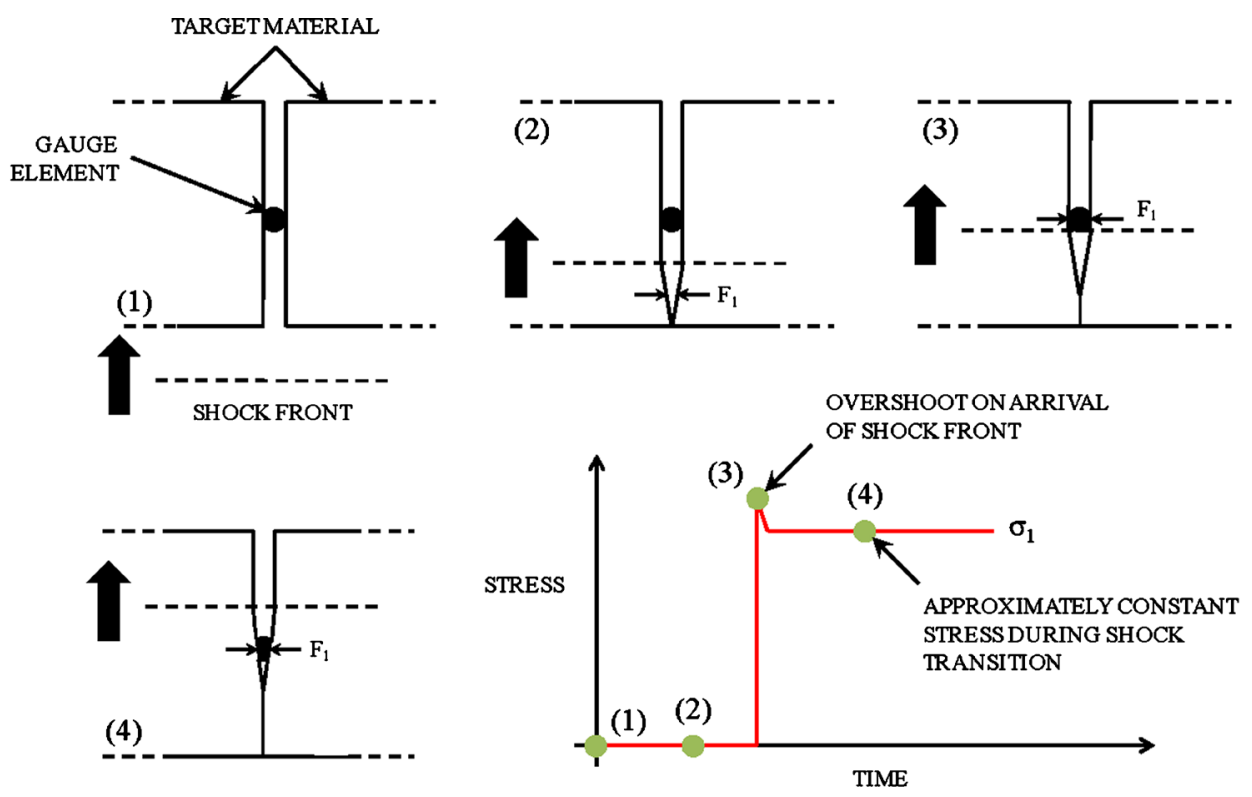

(a) dry-joint configuration
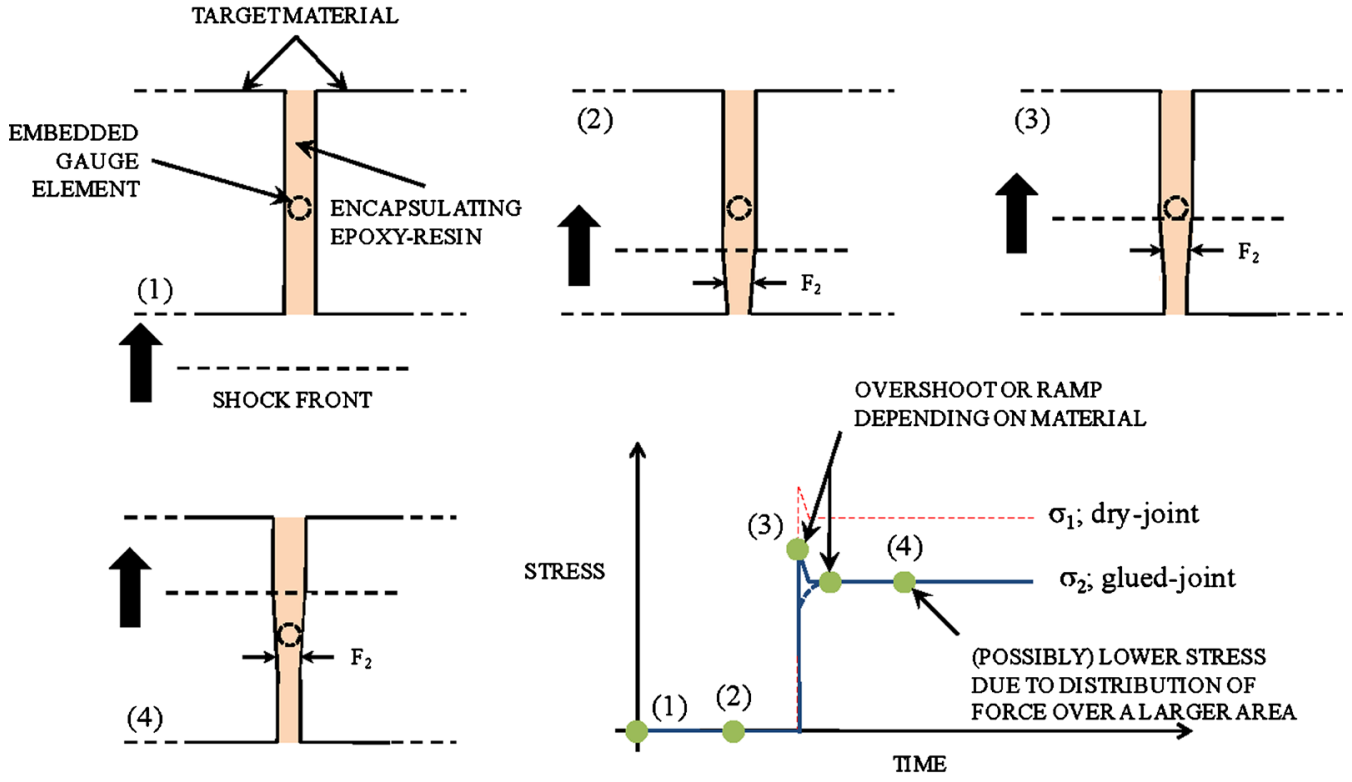

(b) glued-joint configuration

FIG. 6. (Color online) Schematic illustration of the effects of gauge encapsulation on shock evolution in lateral gauge targets.

Winter and Harris ${ }^{10}$ and Winter et al. ${ }^{11}$ Essentially, in the dry-jointed targets the lack of an encapsulation medium would have prevented formation of a propagating shock in the encapsulation region, potentially leading to formation of a Mach stem ${ }^{10,11}$ and subsequent overshoot in stress. However, this explanation is inconsistent with the RTM 6 results where a glued-joint was employed. In these cases, despite similar RTM 6/epoxy encapsulation shock velocities, ${ }^{3,13}$ a gentle ramp-up to a peak stress occurs. The divergent-shock explanation implies that this is caused by a shock running ahead in the encapsulation and a consequent increase in lateral stress following shock arrival. Given the observed nega- tive gradient behind the shock and the similar shock impedances of the target and encapsulation another explanation was considered. It is postulated that the larger gaugeelement-to-target-material surface contact area when an encapsulating interlayer is present acts to redistribute the forces acting on the gauge. This would then lead to a slower ramp-up to a (possibly) lower peak stress. This cushioning concept is illustrated schematically in Fig. 6; arbitrary dry and glued-joint lateral gauge arrangements (neglecting the backing material) are shown in parts (a) and (b), respectively. The different gauge/shock front interaction scenarios illustrated are labeled (1) to (4). A schematic plot illustrating key 
TABLE II. Experimental conditions for cast target material (lard) shots.

\begin{tabular}{|c|c|c|c|c|c|}
\hline Material & $\begin{array}{l}\text { Gauge position } \\
\text { (mm) }\end{array}$ & Mylar position & $\begin{array}{l}\text { Impact velocity } \\
(\mathrm{m} / \mathrm{s})\end{array}$ & $\begin{array}{l}\text { Cu flyer thickness } \\
(\mathrm{mm})\end{array}$ & $\begin{array}{c}\text { Gradient measured } \\
\text { behind shock } \\
\text { between yellow markers } \\
\text { in Fig. } 7 \\
(\mathrm{~V} / \mu \mathrm{s})\end{array}$ \\
\hline Cooperative lard & 4 & Level with front $\mathrm{Cu}$ plate & 194 & 10 & -0.00151 \\
\hline Cooperative lard & 6 & Level with end of encapsulated gauge & 405 & 10 & -0.00345 \\
\hline
\end{tabular}

elements of the gauge response for both dry and gluedinterlayer configurations is included in Fig. 6(b). Shock arrival occurs at point (3); with zero stress at points (1) and (2) beforehand. In the dry-joint case an overshoot occurs as the gauge rings up before equilibrium is subsequently established-e.g., by point (4). When an interlayer is present, however, the overshoot is less severe or even nonexistent (e.g., Fig. 5); with both phenomena illustrated in Fig. 6(b). Effectively, a more gradual gauge loading as the shock front arrives results compared to a virtually instantaneous response in the dry-joint case. In both cases, continued shock propagation, assuming a square input-pulse, will result in a constant rate of compression. Neglecting strengthening effects, a constant stress behind the shock will result (4). It is worth noting that in addition to their link to the nature of gauge encapsulation the initial behavior where an encapsulating layer is employed does appear to have a material-specific component. In particular, the small overshoot in stress in the case of polystyrene (Fig. 4) contrasts with the gentler ramp-up with RTM 6 (Fig. 5), despite both materials exhibiting similar behavior behind the shock. This behavior appears to be indicative of a material-dependant ring-up on shock arrival. Despite this material-specific component, overall these results appear to suggest a link between initial gauge response and the nature of the gauge environment. Consequently, in an attempt to provide further evidence of the true nature of these overshoots in stress and to eliminate any remaining questions arising due to the continued presence of an interlayer region (albeit it without any encapsulating material apart from air) in the dry-joint tests, a further set of experiments involving gauges cast in situ in a target material were carried out.

\section{B. Cast targets}

As discussed above the overshot in stress observed on shock arrival in the dry-joint case for both polystyrene and RTM 6 (Figs. 4 and 5, respectively) had two possible explanations. These were (1) evidence of a Mach stem formed due to the lack of a propagating shock in the empty interlayer ${ }^{10,11}$ or (2) a function of the nature of encapsulation, with the lack of an interlayer in the dry-jointed targets leading to sudden compression on shock arrival at the gauge and, consequently, an overshoot in stress (e.g., Fig. 6). In an attempt to distinguish between these phenomena a series of experiments were conducted involving manganin lateral gauges suspended within a castable target material. This approach, as illustrated schematically in Fig. 2, allowed gauges to be suspended be- tween 4 and $6 \mathrm{~mm}$ from the impact face. Two arrangements were considered. In the first, as shown in Fig. 2, care was taken to ensure that there was $\leq 1 \mathrm{~mm}$ of encapsulating Mylar (designed to enhance gauge stiffness/ensure alignment) ahead of the gauge element. While some Mylar was present ahead of the gauge element the position of this material never moved beyond the end of the gauges employed. In other words, the material ahead of the gauge comprised the as-cast material. It therefore seemed reasonable to suggest that any observed overshoot/changes in lateral stress behind the shock in this test would be attributable to a material response rather than shock dispersion in the virtually nonexistent encapsulating layer. With the second arrangement Mylar was allowed to pass beyond the end of the encapsulated gauge all the way up to the $\mathrm{Cu}$ cover plate shown in Fig. 2, providing a direct comparison to the glue/dry-jointed configurations considered previously. As mentioned earlier in this paper, a commercially available fat (manufactured by Matthews Foods plc and retailed by the Co-operative ${ }^{\circledR}$ Food Group) was employed as the castable target material. This material, due to the complex long-chain structural nature of the constituent glycerol units/attached fatty acids ${ }^{17}$ (analogous to a long-chain polymeric material), was expected to exhibit similar behavior to the polymers studied elsewhere in this paper. As these experiments were only designed to provide a comparison to the response of polystyrene/RTM 6, presentation of results from a small number of tests was judged sufficient to identify any qualitative behavior behind the shock in this castable material. Consequently, results from three tests are included here, with experimental conditions outlined in Table II.

Lateral gauge traces showing the variation in measured voltage with time for the three tests detailed in Table II are presented in Fig. 7. As discussed, this data is uncalibrated due to the previously highlighted issues in measurement of shear wave velocities in the adipose material under consideration. However, the general features of the traces-e.g. gradients and relative magnitudes (allowing for small errors due to the elastic-plastic response of manganin)—would be mirrored if calibrated to show lateral stresses directly. As shown in Fig. 7, in all cases the voltage on shock arrival had not quite reached the calibrated zero-value. This simply means, however, that while data following shock arrival will be essentially proportional to the actual lateral stress, this will not be the case for any preceding data.

There are a number of key features to note in the three traces presented in Fig. 7. At point (a) a ramp-up and subsequent overshoot in stress following shock arrival is apparent 


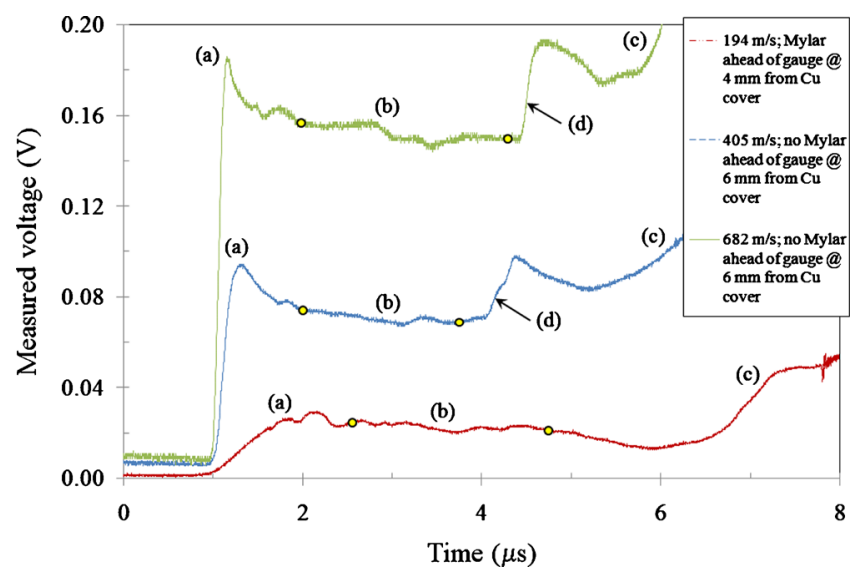

FIG. 7. (Color online) Lateral gauge traces showing the variation in recorded voltage with time for $10 \mathrm{~mm}$ thick $\mathrm{Cu}$ projectiles impacting the arrangement shown schematically in Fig. 2 at three different impact velocities.

on each trace. Significantly the gradient of the initial ramp and the magnitude of the subsequent overshoot increases with impact velocity/pressure. The slower ring-up time in the $194 \mathrm{~m} / \mathrm{s}$ case may be partially attributable to the presence of Mylar ahead of the gauge. However, as the ring-up time will depend on shock velocity in the gauge package-itself a function of pressure-as observed elsewhere, ${ }^{2,3,16,23}$ it follows that a higher velocity shot will lead to a faster ring-up to maximum stress. The continued presence of an overshoot even where no encapsulation is present ahead of the in situ gauge, meaning that minimal/no shock dispersion will result, might appear to favor the geometric argument put forward in Fig. 6. Essentially, this would imply that the overshoot arises due to the temporal nature of shock arrival at the gauge. For example, this would suggest that for the $194 \mathrm{~m} / \mathrm{s}$ shot in Fig. 7 the shock arrives relatively slowly leading to a minimal overshoot, whereas for the $405 \mathrm{~m} / \mathrm{s}$ shot the faster associated wave speed leads to a faster shock arrival and a morepronounced gauge response (analogous to the dry-joint case in Fig. 6). However, following this argument cushioning is likely to have occurred minimising the magnitude of the overshoot as shown for polystyrene in Fig. 4 and in line with this concept if it were possible to include a dry-joint into the lard overshoots of greater magnitude might therefore be expected. However the inclusion of such an air-gap ahead of the gauge is not immediately practical in such an as-cast material. Therefore, it appears that there is insufficient information present within the three traces shown in Fig. 7 to quantatively judge the effect of the presence, or lack thereof, of Mylar ahead of the gauge on this overshoot. Following the initial overshoot negative gradients in recorded voltage are apparent_-point (b) — in all three traces. Measured gradients between the yellow circles indicated in Fig. 7 (recorded in Table II) were $-0.00418 \mathrm{~V} / \mu \mathrm{s},-0.00345 \mathrm{~V} / \mu \mathrm{s}$ and $-0.00151 \mathrm{~V} / \mu \mathrm{s}$ for the $682 \mathrm{~m} / \mathrm{s}, 405 \mathrm{~m} / \mathrm{s}$ and $194 \mathrm{~m} / \mathrm{s}$ shots respectively. While uncalibrated, stress is proportional to measured voltage (albeit with a required allowance for the elastic-plastic response of manganin). Therefore, this increase in the magnitude of the negative gradient with impact stress is, in line with Eq. (1), strongly suggestive of a strengthening phenomena. Similar phenomena were observed previously in this paper for polystyrene and RTM 6 as well as elsewhere for other materials ${ }^{2,7,14-16}$ (although, it should be noted that, while widely accepted and extensive, this work is largely attributable to a limited number of authors). Such a response might be expected given the similarity between the structure (e.g., long polymeric-type chains) of the glycerol units/attached fatty acids which form the adipose material (lard) ${ }^{17}$ in question and the polymeric materials considered previously. Consequently, as with other polymeric materials, the negative gradient observed in Fig. 7 (e.g., strengthening behind the shock) is likely attributable to a steric interference effect. ${ }^{14}$ Finally, at point (c) on all three traces presented in Fig. 7, release waves from the rear of the flyer lead to the end of 1D conditions at the manganin gauge, ending the shock. In addition on the 682 and $405 \mathrm{~m} / \mathrm{s}$ traces at point (d) a reloading event occurs due to arrival of a reflected shock from the rear-surface of the lard target. This occurs because the gauge is located $6 \mathrm{~mm}$ from the $\mathrm{Cu}$ front cover in these two experiments-e.g., just $4 \mathrm{~mm}$ from the rear PMMA (which has a higher density at $1.186 \mathrm{~g} / \mathrm{cm}^{3}$ than the lard ${ }^{22}$ ). Conversely, with the $194 \mathrm{~m} / \mathrm{s}$ shot the gauge is positioned a further $2 \mathrm{~mm}$ from the PMMA, providing sufficient time for releases from the rear of the flyer to catch up before a reflected shock from the PMMA can arrive.

Overall there appears to be a good correlation between the three traces presented in Fig. 7, independent of whether Mylar is present ahead of the gauge or not, with differences directly attributable to the differing impact conditions. A key point appears to be the presence of both an initial overshoot and gradient behind the shock (in this case in recorded voltage) in the 682 and $405 \mathrm{~m} / \mathrm{s}$ cases. Both the overshoot and gradient are of a greater magnitude than the corresponding features in the $194 \mathrm{~m} / \mathrm{s}$ case, despite the lack of Mylar ahead of the gauge which would negate shock dispersion and the associated stress perturbations/potential Mach stem formation. Given that gauges are cast in situ this result seems to suggest that lateral gauge response is consistent independent of the presence or otherwise of an encapsulating Mylar layer; e.g., that the embedded gauge is, at least to some extent, detecting a true material response. This conclusion backs the evidence of a lack of an interlayer effect on gradient behind the shock inferred from the similarity of glued and dryjointed lateral gauge traces in a particular material presented earlier. Further, it is also supported in the literature; e.g., the presence of similar gradients on in situ cast gauges observed by Millett et al. in the elastomeric material Sylgard. ${ }^{14}$

\section{CONCLUSIONS}

Plate-impact experiments have been employed on polymeric-like materials in three different arrangements to investigate the nature of lateral manganin stress gauge response. In the first set of tests the response of lateral gauges in simple and complex polymeric materials (polystyrene and RTM 6, respectively) was studied employing both an encapsulating Mylar/epoxy interlayer and a dry-joint with no interlayer present. The nature of the encapsulating layer has been shown to influence initial response and the ramp-up to 
maximum stress recorded by lateral gauges. Significant overshoots in stress following shock arrival were recorded where a dry-joint was employed, not present in the encapsulatedgauge case. This was attributed to a cushioning effect, where the presence of an interlayer led to redistribution of force away from the embedded gauge element. Given that a dryjoint arrangement arguably detects a material response-only, this result does suggest that, at least during the early part of the shock, allowance for the presence of an interlayer in typical embedded lateral gauge target geometries may be required. However, further work is clearly required before any firm conclusions can be reached. Finally, in otherwise essentially identical experiments, constant gradients behind the shock independent of the nature of gauge encapsulation, but dependant on the target material have been observed. Taken together these modified-interlayer results appear suggestive of detection of a material-based response by embedded lateral gauges behind the shock.

Similar conclusions were reached where a lateral gauge was directly embedded in an adipose material (Matthews Foods plc/Co-operative ${ }^{\circledR}$ Food Group "Fresh Fields Lard"). Insufficient information was available to definitively identify the cause of observed overshoots following shock arrival, although there was tentative evidence that the geometric effect postulated to explain similar behavior observed in the dry-glued-joint polystyrene lateral responses was involved. Significantly, even when the gauge was almost entirely isolated within the as-cast material (e.g., $\leq 1 \mathrm{~mm}$ of Mylar protruded ahead of the gauge element) a substantial gradient was apparent behind the shock. Given that the lack of an interlayer would prevent shock dispersion this appeared to be indicative of a hardening phenomena.

Overall, these results-particularly the consistent nature of gradients behind the shock for a given material when the encapsulating interlayer was substantially modified-appear to suggest that, at least in polymeric systems, changes in lateral gauge trace gradient behind the shock are related to a material response rather than a geometric target-construction effect.

\section{ACKNOWLEDGMENTS}

The Institute of Shock Physics (Imperial College London) is gratefully acknowledged for their sponsorship of this research program. We are also indebted to Mr. Andy Roberts for associated technical support. British Crown Owned Copyright 2010/MOD.

${ }^{1}$ J. E. Field, S. M. Walley, W. G. Proud, H. T. Goldrein, and C. R. Siviour, Int. J. Impact Eng. 30, 725 (2004).

${ }^{2}$ G. J. Appleby-Thomas, P. J. Hazell, C. Stennett, G. Cooper, K. Helaar, and A. M. Diederen, J. Appl. Phys. 105, 064916 (2009).

${ }^{3}$ G. J. Appleby-Thomas, P. J. Hazell, and C. Stennett, J. Mater. Sci. 44, 6187 (2009)

${ }^{4}$ M. A. Meyers, Dynamic Behaviour of Materials (Wiley, New York, 1994).

${ }^{5}$ J. C. F. Millett, N. K. Bourne, G. T. Gray III, and G. Cooper, AIP Conf. Proc. 620, 131 (2002).

${ }^{6}$ N. K. Bourne and J. C. F. Millett, Proc. R. Soc. London, Ser. A 459, 567 (2003).

${ }^{7}$ J. C. F. Millett, N. K. Bourne, and N. R. Barnes, J. Appl. Phys. 92, 6590 (2002).

${ }^{8}$ J. C. F. Millett, N. K. Bourne, and D. P. Dandekar, J. Appl. Phys. 96, 3727 (2004).

${ }^{9}$ G. T. Gray III, N. K. Bourne, and J. C. F. Millett, J. Appl. Phys. 94, 6430 (2003).

${ }^{10}$ R. E. Winter and E. J. Harris, J. Phys. D: Appl. Phys. 41, 035503 (2008). ${ }^{11}$ R. E. Winter, G. D. Owen, and E. J. Harris, J. Phys. D: Appl. Phys. 41, 202006 (2008).

${ }^{12}$ J. C. F. Millett, G. T. Gray III, and N. K. Bourne, J. Appl. Phys. 101, 033520 (2007).

${ }^{13}$ P. J. Hazell, C. Stennett, and G. Cooper, Polym. Compos. 29, 1106 (2008).

${ }^{14}$ J. C. F. Millett, G. Whiteman, S. M. Stirk, and N. K. Bourne, Society for Experimental Mechanics (SEM) Annual Conference and Exposition on Experimental and Applied Mechanics, 1-4 June, 2009, Albuquerque, NM, p. 498.

${ }^{15}$ J. C. F. Millett and N. K. Bourne, J. Phys. D: Appl. Phys. 37, 2901 (2004).

${ }^{16}$ C. E. Tyler, N. K. Bourne, and J. C. F. Millett, AIP Conf. Proc. 955, 687 (2007).

${ }^{17}$ B. R. Mackenna and R. Callander, Illustrated Physiology (Churchill Livingstone, New York, 1990).

${ }^{18}$ N. K. Bourne, Meas. Sci. Technol. 14, 273 (2003).

${ }^{19}$ Z. Rosenberg and Y. Partom, J. Appl. Phys. 58, 3072 (1985)

${ }^{20}$ Z. Rosenberg and N. S. Brar, J. Appl. Phys. 77, 1443 (1995).

${ }^{21}$ J. C. F. Millett, N. K. Bourne, and Z. Rosenberg, J. Phys. D: Appl. Phys. 29, 2466 (1996).

${ }^{22}$ S. P. Marsh, LASL Shock Hugoniot Data (University of California Press, Los Angeles, CA, 1980)

${ }^{23}$ G. J. Appleby-Thomas, P. J. Hazell, J. Millett, and N. K. Bourne, AIP Conf. Proc. 1195, 533 (2009). 